\title{
A Double Take at Conferences: The Hybrid Format
}

\author{
Anni-Yasmin Turhan ${ }^{1}$
}

Published online: 18 February 2022

(c) The Author(s), under exclusive licence to Springer-Verlag GmbH Germany and Gesellschaft für Informatik e.V. 2022

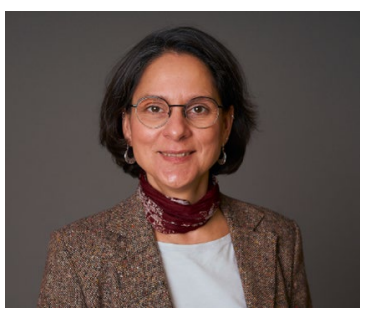

Dear readers,

Conferences for the AI community are important events to disseminate latest scientific results and to anticipate and discuss upcoming trends in the wider research field. In the first year of the Covid pandemic conference organizers had to implement an all on-line conference format as more of an emergency solution. The more recent editions of many conferences were conducted in a hybrid format where some of the participants joined physically on-site and some virtually on-line.

The on-line conference format certainly has its benefits as it is flexible, it has a small impact on the environment due to reduced travels and it is more inclusive than face to face conferences, since the registration costs are lower and there would be no visa problems for attendees. Moreover, communication between the participants need not be synchronous anymore, as talks can be recorded and made available for a longer period. This alleviates to a certain extend the problem that participants attend from different time zones.

However, the switch to online versions was not merely about how to deliver talks, panel sessions and discussions to the participants, but also about how to replicate settings for the social interaction that are usually taking place at face to face conferences. The direct, personal conversations with colleagues is what makes attending a conference fruitful, interesting, and motivating. In fact, not few attendees find that the coffee breaks are one of the most rewarding parts of conferences for exactly that reason. The need for direct

Anni-Yasmin Turhan

anni-yasmin.turhan@tu-dresden.de

1 Department of Computer Science, Dresden University of Technology, Dresden, Germany interaction is even more necessary for a wide and diverse field as AI is. With the new challenges that our field is currently facing and with its impact on society and the environment, a further splitting and isolation into sub-communities is not desirable.

To replicate the setting for social interaction that face to face conferences offer in the on-line version, turned out hard to achieve. This is not merely due to the technical support, but also due to several other effects. After two years of social distancing, we all tend to suffer from on-line fatigue and the increased work load due to the pandemic. This lets attendees visit on-line events sparingly. Browsing through talks in sessions outside of the own working field seems to be expendable - an assessment which may deprive scientists of the chance to discover new connections. Furthermore, online social events can be a fairly uneasy experience. Recent surveys ${ }^{1}$ indicate that a pure on-line conference format is the least favorite compared to face to face and hybrid conferences. Even remotely participating attendees reported that social interaction feels more natural in a hybrid setting. The need for real-time social interaction among the participants made the hybrid format the favored choice.

Some of the issues with on-line conferences also persist in this format. As the participants are located in different time zones, the choice of a "reference time zone" and the preparation of the conference schedule remains a non-trivial task. Here, the often adopted shortening of the sessions leads to very dense schedules. This not necessarily helping attendees to get most out of the conference. An underlying problem here appears to be that on-line conferences do not per se become the main focus of the participant's attention for several days as face to face conferences can be. It seems harder to signal unavailability for the whole time of a conference, if no travel is involved. The temptation to engage in an on-line conference only session-wise is big. Nevertheless,

\footnotetext{
1 e.g. https://www.frontiersin.org/articles/10.3389/fmars.2021. 638025/full.
} 
the context change and the opportunity to immerse in a conference is important to concentrate on the new ideas presented and to discuss them with colleagues. Here, it would be helpful to establish the norm that participating in an online or hybrid conference means to make time for the whole event and to be able to attend as many sessions and events as offered.

Recently, there were somewhat new forms of organizing and conducting conferences conducted in order to foster personal interactions while implementing social distancing. For instance, in 2021 AAAI had repeating poster sessions that alleviated the time zone problem and gave more room for interaction. An approach to address the travel dilemma is to organize a hybrid conference with several physical hubs. This was, for example, adopted by KR last year and seems to become a more popular approach to strengthen the opportunity for physical meetings.

The long term effects of the hybrid conferences still need to play out, but the scientific community needs to decide on what to keep and what to let go of. The search for features that encourage and foster direct connection between on-line (and off-line) participants is on-going. In any case, the hybrid format offers a variety of opportunities to try out new variants. We should keep experimenting and build on the early experiences being already made.

Best wishes and enjoy reading this issue of $\mathrm{KI}$,

$$
\text { Ami-yasmir Jushar }
$$

Anni-Yasmin Turhan

\section{Forthcoming Special Issues}

\subsection{Application of Al in Digital Forensics}

Guest Editors: Johannes Fähndrich, Roman Povalej, Heiko Rittelmeier, Silvio Berner

Scope: With the increase of digitalization and the pervasiveness of information systems, a crime scene is no longer what it used to be with its mix of a location, people, evidence, changes in time, and their virtual counterpart. Including the mainstream use of smart-homes, -infrastructure, -factories, or -cities, investigations and forensic evidence are no longer bound by physics. With the growing amount of digital information, an application of Artificial Intelligence (AI) in forensics is incumbent. Methods from Machine Learning and Data Science need to be extended to be explainable and valid for legal purposes. This special issue has the goal of collecting work on AI with the application on forensic science with the focus on the amalgamation of computer science, data analytics, and machine learning with the discussion of the law and ethics for its application to cyber forensics.

Topic might be, but are not restricted to:

NLU/NLP in forensic evidence

Explainable AI which can stand up in court

AI and object detection

AI and super resolution

$\mathrm{AI}$ and darknets and hidden services investigation

$\mathrm{AI}$ and emotion recognition

$\mathrm{AI}$ and lie detection

AI and cybercrime related investigations

- Fooling neuronal networks and other anti-forensic techniques and methods automated analysis for forensic evidence in IoT

AI in incident response, investigation and evidence handling

Ethical, legal, and societal challenges of using AI in digital forensics

Contact: Johannes Fähndrich

(johannesfaehndrich@hfpol-bw.de)

\subsection{Explainable AI}

Guest Editors: Ute Schmid (Universität Bamberg), Britta Wrede (Universität Bielefeld)

Scope: During the last years, Explainable AI (XAI) has been established as a new area of research focusing on approaches which allow humans to comprehend and possibly control machine learned (ML) models and other AI-systems whose complexity makes the process which leads to a specific decision intransparent. In the beginning, most approaches were concerned with post-hoc explanations for classification decisions of deep learning architectures, especially for image classification. Furthermore, a growing number of empirical studies addressed effects of explanations on trust in and acceptability of AI/ML systems. Recent work has broadened the perspective of XAI, covering topics such as verbal explanations, explanations by prototypes and contrastive explanations, combining explanations and interactive machine learning, multi-step 
explanations, explanations in the context of machine teaching, relations between interpretable approaches of machine learning and post-hoc explanations, neuro-symbolic approaches and other hybrid approaches combining reasoning and learning for XAI. Addressing criticism regarding missing adaptivity more interactive accounts have been developed to take individual differences into account. Also, the question of evaluation beyond mere batch testing has come into focus.

In the special issue, the focus will be on research addressing such recent developments in XAI. Furthermore, interdisciplinary contributions as well as specific applications of XAI form domains such as education, healthcare, and industrial production are welcome.

The topics of interest for the special issue include, but are not limited to:

interactive approaches to XAI

adaptive XAI

- deployment of explainable decision support systems in real-life settings (e.g. medical domain, work contexts etc.)

multi-modal approaches to XAI

process explanations

self-explaining robots

empirical evaluation of XAI approaches

measures for understanding of XAI

evaluation measures for XAI beyond trust and acceptability Contributions can be from the following categories (for more detailed information please refer to the author instructions for each of these categories): Technical Contribution; System Descriptions; Project Reports; Dissertation and Habilitation Abstracts; AI Transfer; Discussion

If you are interested in submitting a paper please contact one of the guest editors:

Contact: Ute Schmidt

(ute.schmid@uni-bamberg.de)

\subsection{GeoAl}

Guest Editors: Simon Scheider, Zena Wood, Kai-Florian Richter
Scope: Researchers in Artificial Intelligence (AI) and Geography have been developing various points of contact in the past, with many possibilities of mutual benefit in the future. Recently, subsymbolic AI methods, such as Deep Learning, have increased the quality and scalability of data processing methods in remote sensing, geographic information retrieval, natural language processing (NLP) and geospatial modeling, among others. Furthermore, there is a tradition of using symbolic AI approaches to raise the quality and scalability of methods by linking, e.g., Geography with agent-based simulation (ABM), spatial cognitive reasoning with Robotics, as well as Geography with the Knowledge Graphs (KG) in the Semantic Web. At the same time, geographic information has become an indispensable resource in itself, needed not only for adding spatial intelligence to machines, and for making opaque models transparent, but also for understanding what kind of intelligence is needed to refer to place and to handle space. Understood in this broader sense, geoAI has the potential of fundamentally improving the way geographic information can be processed and interpreted by both humans and machines.

For this special issue, we invite researchers who investigate the kind of knowledge needed to account for Geography and space with(in) intelligent machines. We are looking for original research articles, project reports and discussion articles on (among others):

Symbolic (Semantic Web and ontological) approaches to geoAI

Sub-symbolic (deep learning/ML) based approaches to geoAI

- Explainable geoAI (XgeoAI): interpreting and opening black box models with a-priori knowledge

Computational models of geospatial intelligence and spatial cognition

Methods for geospatial knowledge graphs (geoKG)

Reusability of geoAI models and reproducibility

Knowledge models of Geography and geographic information for data scientists

- Pragmatic intelligence: Models of purpose and design of workflows with geoinformation

The human in the loop and models of human interaction in geoAI

Application areas include, but are not restricted to:

Agent-based models (ABM) and geoAI in Geography and Geosciences 
- $\mathrm{AI}$ in geographic information retrieval (GIR) and NLP: distant reading of geolocated texts

Geographic question-answering (geoQA) and automation of geographic data analysis

AI-enhanced geovisualization and dialogue methods

Object recognition in remote sensing and georeferenced image processing
geoAI in robotics, ubiquitous sensors and navigation systems

Contacts: Simon Scheider (s.scheider@uu.nl),

Zena Wood (Z.M.Wood2@exeter.ac.uk),

Kai-Florian Richter (kai-florian.richter@umu.se) 